\title{
Mid-wave Infrared Quantum Dot Quantum Cascade Photodetector Monolithically Grown on Silicon Substrate
}

\author{
Jian Huang,* Daqian Guo,* Zhuo Deng, Wei Chen, Huiyun Liu, Jiang Wu, and Baile Chen
}

\begin{abstract}
Mid-infrared photodetector based on sub-monolayer (SML) quantum dot quantum cascade structure monolithically grown on silicon substrate has been demonstrated in this work. Both the optical and electrical characteristics of the SML quantum dot quantum cascade photodetectors (QD-QCD) were analyzed quantitatively. The performances of these devices were compared with that on native GaAs substrate. A large resistance-area $\left(R_{0} A\right)$ product of $1.13 \times 10^{7} \Omega . \mathrm{cm}^{2}$ is achieved at 77 $K$ for the silicon based devices, which is only roughly one order less than that on GaAs substrate. The device shows a normal-incident peak responsivity of $0.59 \mathrm{~mA} / \mathrm{W}$ under zero bias at the wavelength of $6.2 \mu \mathrm{m}$ at $77 \mathrm{~K}$, indicating a photovoltaic operation mode. Johnson noise limited specific detectivity is $3 \times 10^{10} \mathrm{~cm} \cdot \mathrm{Hz}^{1 / 2} / \mathrm{W}$ at $77 \mathrm{~K}$, with photoresponse up to $100 \mathrm{~K}$. These results suggest that the silicon-based QD-QCD in this work is a very promising candidate for large format mid-infrared focal plane array and mid-infrared silicon photonics applications.
\end{abstract}

Index Terms-infrared photodetector, quantum cascade, quantum dots, silicon substrate.

\section{INTRODUCTION}

$\mathrm{M}$ ID-WAVE infrared (MWIR) detectors have attracted great attention in many applications such as chemical fingerprinting, gas monitoring and high-performance infrared imaging. Inter-subband infrared photodetectors like quantum cascade detectors (QCDs) and quantum well infrared photodetectors (QWIPs) are of great interesting in last several decades, due to their coverage of the MWIR spectral region and high band structure engineering freedom in operational wavelength.

Over the past several years, much progress on the development of high performance quantum cascade

This work was supported in part by the Shanghai Sailing Program under Grant 17YF1429300, in part by the ShanghaiTech University startup funding under Grant F-0203-16-002 and in part by the UK EPSRC First Grant EP/R006172/1. (Corresponding authors: Jiang Wu and Baile Chen.)

J. Huang, Z. Deng, W. Chen and B. Chen are with the Optoelectronic Device Laboratory, School of Information Science and Technology, ShanghaiTech University, Shanghai 201210, Peoples R China (e-mail: huangiian@shanghaitech.edu.cn; $\quad$ dengzhuo@shanghaitech.edu.cn; chenwei@ shanghaitech.edu.cn; chenbl@ shanghaitech.edu.cn ).

D. Guo, H. Liu and J. Wu are with the Department of Electronic and Electrical Engineering, University College London, London WC1E 7JE, United Kingdom (e-mail: daqian.guo.15@ucl.ac.uk; huiyun.liu@ucl.ac.uk; jiang.wu@ucl.ac.uk ).

*These authors contributed equally to this work. photodetectors has been made [1-3]. These devices enjoy the advantage of zero bias operation, lower dark current, high operating temperature and small power dissipation [4]. It is known that the infrared light absorption in quantum cascade photodetectors are generally based on inter-subband transition in the quantum well structures. Therefore, one disadvantage of quantum well based QCD (QW-QCD) is that these devices have no intrinsic response to normal incidence light, due to the inter-subband transition selection rules in quantum wells. Recently, one variation of the QW-QCD has been demonstrated, which uses the quantum dots instead of quantum wells as light absorption region [5-7]. That is very similar to the case of quantum dot infrared photodetectors (QDIP) versus quantum well infrared photodetectors (QWIP). For the first time, Barve et al. reported a GaAs-based Stranski-Krastanov (SK) mode quantum dot QCD (QD-QCD), with optical response peaks between 5 to $6 \mu \mathrm{m}$ [5]. Wang et al. reported a QD-QCD grown on InP substrate which enjoys the abundance of semiconductor materials grown on InP substrate [7]. Recently, our group reported a GaAs-based sub-monolayer QD-QCD (SML QD-QCD), with optical response peaks around $6 \mu \mathrm{m}$ [6].Both of these QD-QCDs have been demonstrated to show normal incident response with low dark current and high detectivity.

Besides fundamental research, the monolithic integration of infrared photodetectors on silicon substrates $[8,9]$ have also received wide attention from the application-oriented communities, due to the following advantages. First, larger area of III-V substrates (such as GaAs, GaSb and InP) are currently under development. Large area and high quality silicon substrates are much cheaper than any III-V substrates. Demonstration of MWIR photodetectors on silicon substrate can significantly reduce the cost of these devices. Moreover, mid-infrared (MIR) silicon photonics is recently attracting increasing attention for possible application in "lab on a chip" technologies for chemical sensing and free space communication [10]. Several passive silicon photonics devices in the MWIR range have been demonstrated [11, 12]. Beside passive components, photodetectors are one of the key components required in the MWIR silicon photonics applications. In this paper, we report a sub-monolayer quantum dot quantum cascade photodetector (SML QD-QCD) monolithically grown on silicon substrate. A responsivity of $0.59 \mathrm{~mA} / \mathrm{W}$ under zero bias at $77 \mathrm{~K}$ is demonstrated, with resistance-area $\left(R_{0} A\right)$ product of $1.13 \times 10^{7} \Omega . \mathrm{cm}^{2}$. The 
absorbing active region of the photodetectors consists of quantum cascade structures, which may allow for integration of quantum cascade laser on the same platform.

\section{DEVICE DESIGN}

The SML QD-QCD samples were grown by using a solid-source Veeco Gen-930 molecular beam epitaxy system. Si (100) substrates with $4^{\circ}$ offcut towards the [011] direction and arsenic pre-layer technique were used to avoid the formation of anti-phase domains. The Si substrates were deoxidized at $900{ }^{\circ} \mathrm{C}$ for 30 minutes. Then, the substrates were cooled down to $370^{\circ} \mathrm{C}$ for the growth of a $5 \mathrm{~nm}$ AlAs nucleation layer through migration enhanced epitaxy. Following the nucleation layer, a $1000 \mathrm{~nm}$ GaAs buffer layer and two sets of dislocation filter layers (DFLs) were grown successively to improve material quality. Each DFL is made of ten periods of $10 \mathrm{~nm}$ $\mathrm{In}_{0.15} \mathrm{Ga}_{0.85} \mathrm{As} / 10 \mathrm{~nm}$ GaAs strained-layer super-lattices and a $350 \mathrm{~nm}$ thick GaAs spacing layer. After the last GaAs spacer, the growth of the detector active region was then carried out as follows: a $400 \mathrm{~nm}$ GaAs bottom contact layer with $\mathrm{Si}$ doped to $1 \times 10^{18} \mathrm{~cm}^{-3}$, a $50 \mathrm{~nm}$ $\mathrm{Al}_{0.07} \mathrm{Ga}_{0.93} \mathrm{As}$ barrier, 20 repeats of SML QDs and quantum cascade quantum wells, and a $50 \mathrm{~nm} \mathrm{Al}_{0.07} \mathrm{Ga}_{0.93} \mathrm{As}$ barrier. The structure and growth conditions of the active region are the same as that of a previous SML-QD QCD reported by us [6]. The active region was finally capped by a Si doped $\left(\mathrm{n}=1 \times 10^{18} \mathrm{~cm}^{-3}\right) 200 \mathrm{~nm}$ top contact. Fig. 1 shows the layer schematics of the SML QD-QCD sample structure and Fig. 2 presents one period of the conduction band of the device with the electronic wavefunction and energy level simulated with $\mathrm{k}$. p method [13-15].

\section{MATERIAL CHARACTERIZATION}

Fig. 3 presents the X-ray diffraction (XRD) Omega-2Theta scan curves of the SML QD-QCD grown on Si substrate. For comparison purpose, the XRD curve recorded from the SML QC-QCD structure grown on GaAs substrate [6] is also presented in the figure. Despite the position and periodicity of the satellite peaks remain unchanged, it is clear that the peak widths of the SML QD-QCD grown on Si significantly broaden compared with that of the GaAs-based sample, indicating some of the dislocation defects may propagate into the active region. This issue should be further optimized in the growth process in our future works, e.g. using more repeats of DFLs.

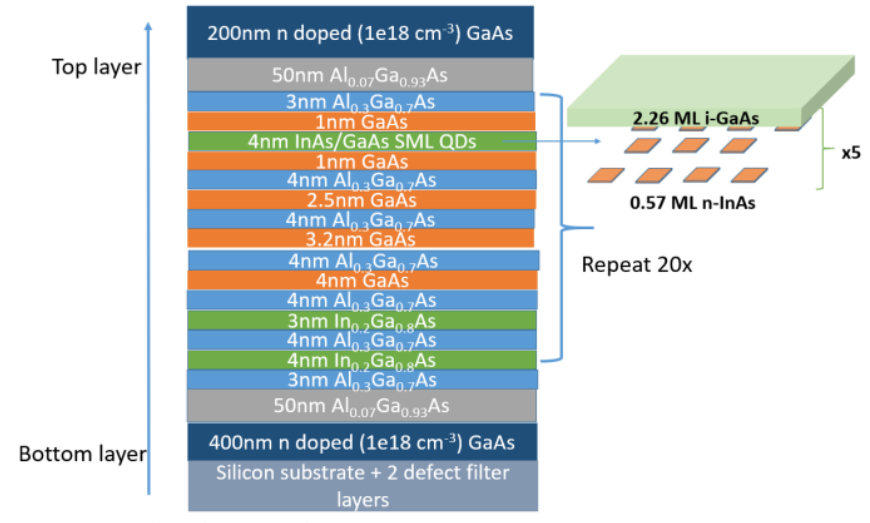

Fig. 1. Schematics diagram of the SML QD-QCD sample structure.

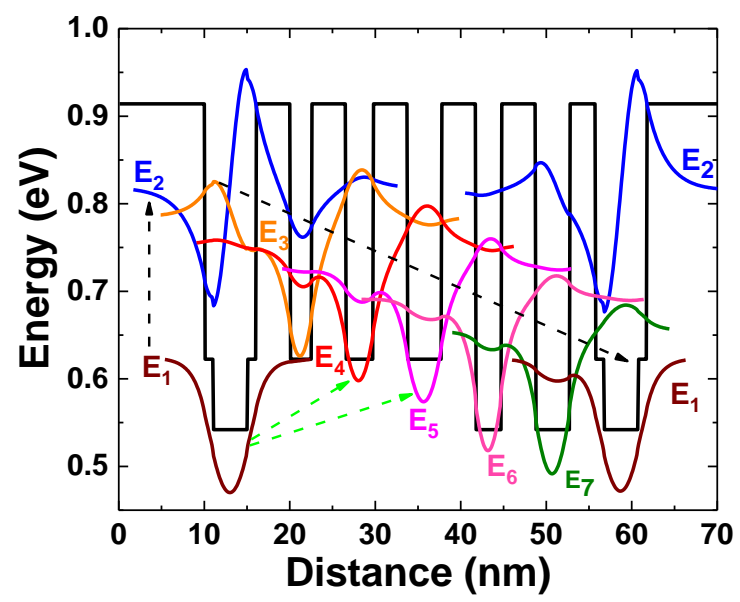

FIG. 2. Conduction band alignment of one period of the SML QD-QCD showing the calculated eigen-levels and electron wave functions. Starting from the left, the layer sequence is as follows: $3 \mathrm{~nm}$ AlGaAs/ $1 \mathrm{~nm} \mathrm{GaAs} / 4 \mathrm{~nm}$ $\mathrm{InGaAs} / 1 \mathrm{~nm} \mathrm{GaAs} / 4 \mathrm{~nm} \mathrm{AlGaAs} / 2.5 \mathrm{~nm} \mathrm{GaAs} / 4 \mathrm{~nm} \mathrm{AlGaAs} / 3.2 \mathrm{~nm} \mathrm{GaAs} / 4$ $\mathrm{nm}$ AlGaAs/4 nm GaAs/4 nm AlGaAs/3 nm InGaAs/4 nm AlGaAs/4 nm InGaAs. The black arrows indicate the electronic path during the detection mode, and the green arrows indicate the diagonal transitions of electrons which account for the dark current.

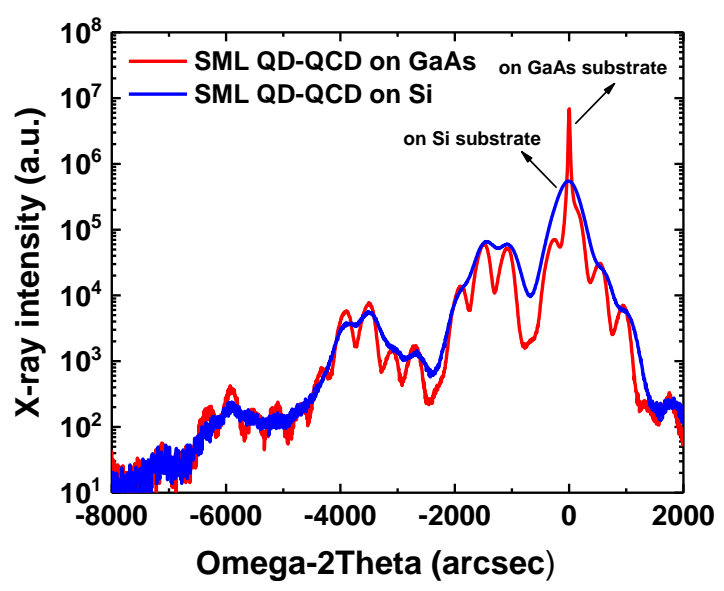

Fig. 3. XRD rocking curves of the SML QD-QCD

The optical properties of the SML QD-QCD sample were studied by the continuous-wave photoluminescence (PL) measurements. The sample was loaded in a variable-temperature closed-cycle helium cryostat, and was excited by the $532 \mathrm{~nm}$ laser. The PL signal from the sample was analyzed by a monochromator and detected by a liquid nitrogen-cooled InGaAs photodiode detector array. For comparison purpose, the temperature-dependent PL spectra of a GaAs-based SML-QD QCD studied in our previous work were also measured. During the measurement the optical setups were kept in optimized conditions for both samples, thus making the direct comparison of PL intensity between two samples meaningful. Fig. 4 shows the temperature-dependent PL spectra of both samples measured from 80 to $300 \mathrm{~K}$ at a fixed 
laser excitation power of $28 \mathrm{~mW}$. Two emission peaks can be observed in both samples. The lower energy emission (labelled as peak A), located around 1.41-1.42 eV at $80 \mathrm{~K}$, could be attributed to the inter-band radiative recombination between the ground states of electron and hole ( $E_{1}$ in Fig. 2) in the SML InAs/GaAs QD regions. The emission of higher energy peak (labelled as peak B) could be associated with the bulk GaAs layers. From Fig. 5, as expected, it can be seen that the PL intensity measured from the Si-based sample is weaker than that of the GaAs-based sample, which is due to the increase of non-radiative recombination caused by the propagation of defects in the active region as seen in the XRD measurement. The peak positions of the two samples are slightly different, which indicates some residual strain left in the Si-based sample. What is even more interesting is that unlike the GaAs-based sample, the FWHM of the quantum dot ground state emission, i.e., peak A, of the Si-based sample does not follow the typical phonon scattering-induced broadening with increasing temperature, as presented in Fig. 5. It remains insensitive to temperature below $160 \mathrm{~K}$, and it shows a rapid decrease when temperature is higher than $160 \mathrm{~K}$. This temperature behavior is indicative of a larger inhomogeneity of quantum dot sizes in the Si-based sample, where such anomalous shift is due to thermal carrier transfer from smaller size quantum dots to the larger size quantum dots with narrower electronic states distribution[16,
17]. Therefore, based on the FWHM comparison between two samples, it can be concluded that the quantum dot sizes in Si-based sample is not as uniform as that in GaAs-based sample.

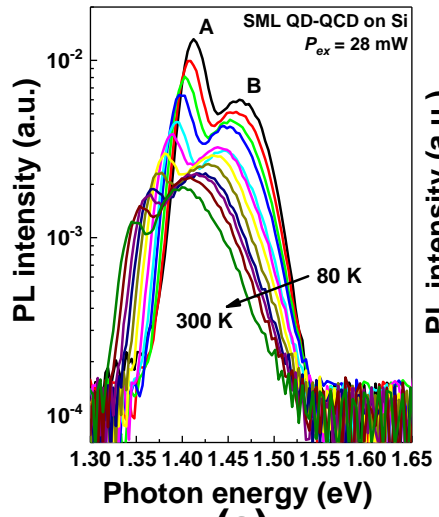

(a)

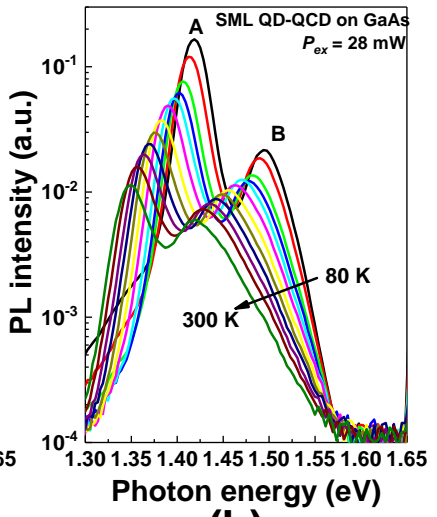

(b)
Fig. 4. Temperature-dependent PL spectra of the SML QD-QCD sample grown on (a) Si substrate and (b) GaAs substrate, measured from 80 to $300 \mathrm{~K}$. The excitation laser power was fixed at $28 \mathrm{~mW}$ in all measurements.

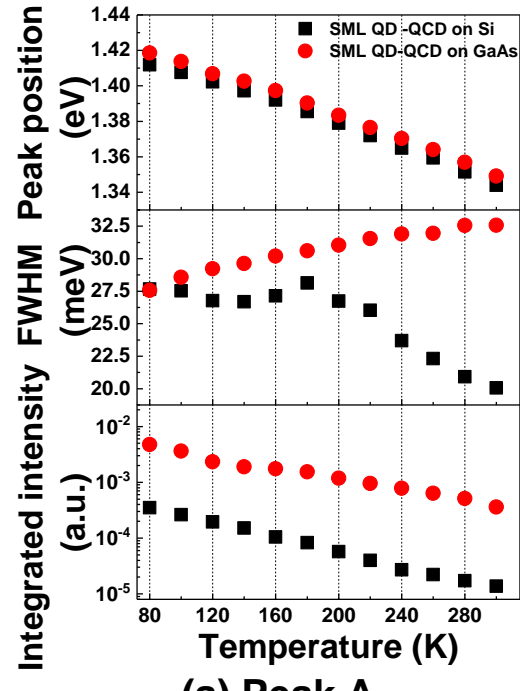

(a) Peak A

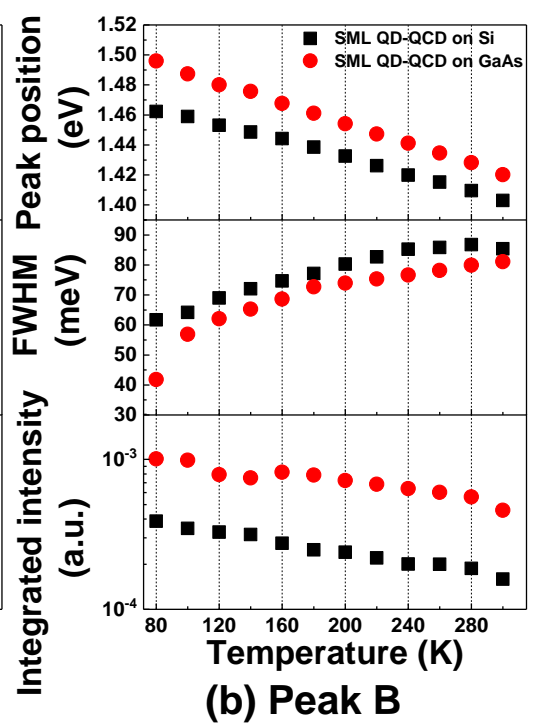

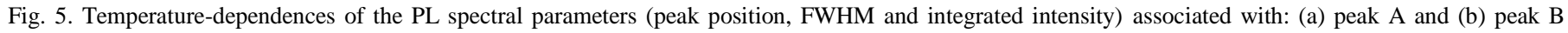
extracted from Fig. 4. Solid squares represent the SML QD-QCD grown on Si, whereas solid circles correspond to the SML QD-QCD grown on GaAs.

\section{DEVICE FABRICATION AND CHARACTERIZATION}

After the crystallographic and optical characterizations, the sample was processed into a set of unpassivated mesa-isolated photodetector devices with diameters ranging from $20 \mu \mathrm{m}$ to $500 \mu \mathrm{m}$. Standard UV photolithography and wet chemical etching methods $\left(\mathrm{H}_{3} \mathrm{PO}_{4}: \mathrm{H}_{2} \mathrm{O}_{2}: \mathrm{H}_{2} \mathrm{O}=1: 1: 8\right)$ were utilized to define the mesa. Metal contacts of Ti/Au $(50 \mathrm{~nm} / 300 \mathrm{~nm})$ were deposited at the top and bottom n-doped $200 \mathrm{~nm}$ GaAs layers by using electron beam evaporation and lift-off techniques.

The temperature dependence of the dark current density-voltage characteristics of a SML QD-QCD sample with $130 \mu \mathrm{m}$ diameter are shown in Fig. 6. The current signal was measured by enclosing the sample in a variable-temperature 
probe station with cold shield covered and recorded by a semiconductor device analyzer. At $77 \mathrm{~K}$, a very low dark current density of $2.11 \times 10^{-8} \mathrm{~A} / \mathrm{cm}^{2}$ is obtained at $-0.1 \mathrm{~V}$ and it increases to $4.12 \times 10^{-5} \mathrm{~A} / \mathrm{cm}^{2}$ at room temperature. The asymmetric nature of the $J-V$ is consistent with the band structure of the photodetector. The dark current density demonstrated in this structure is evidently lower than that in the QD-QCD reported in Barve 's [5] work and Gautam's [18] work. This low value of dark current can also be revealed in the resistance area product $\left(R_{0} A\right)$ of the device as a function of temperature as shown in Fig. 7. At $77 \mathrm{~K}$, the $R_{0} A$ has a value of $1.13 \times 10^{7} \Omega . \mathrm{cm}^{2}$, and decreases to $1476 \Omega . \mathrm{cm}^{2}$ at $300 \mathrm{~K}$, indicating a very low Johnson noise in the device. From this Arrhenius plot the activation energy $\left(E_{a}\right)$ can be estimated. The linear fits yield an activation energy of $285 \mathrm{meV}$ at high temperature region. Given the band structure of the detector, this value of activation energy can be interpreted as the average thermal energy required for conduction which associated with the current leakage paths from $E_{1}$ to $E_{2}$ and $E_{1}$ to continuous state above the AlGaAs barriers as shown in Fig. 2 [19] . In low temperature region, $E_{a}$ decreases to $40.9 \mathrm{meV}$, and this activation energy could be explained as follows: the dark current generally involves several diagonal transitions from one cascade to the next and the transitions contributing to dark current are highly temperature dependent. As the temperature decreases, the transition rate of electronic transition between two consecutive cascades increases and dominates, which causes the dark current being dominated by the electronic diagonal transitions from level $\mathrm{E}_{1}$ to $\mathrm{E}_{4}$ or $\mathrm{E}_{5}$ as shown in Fig. 2 $[20,21]$.

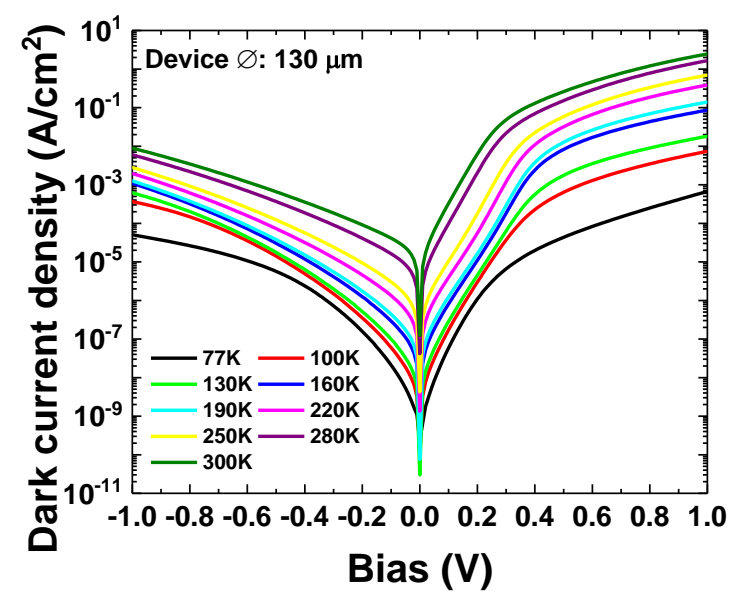

Fig. 6. Dark current density as a function of bias measured at different temperatures. The device diameter is $130 \mu \mathrm{m}$.

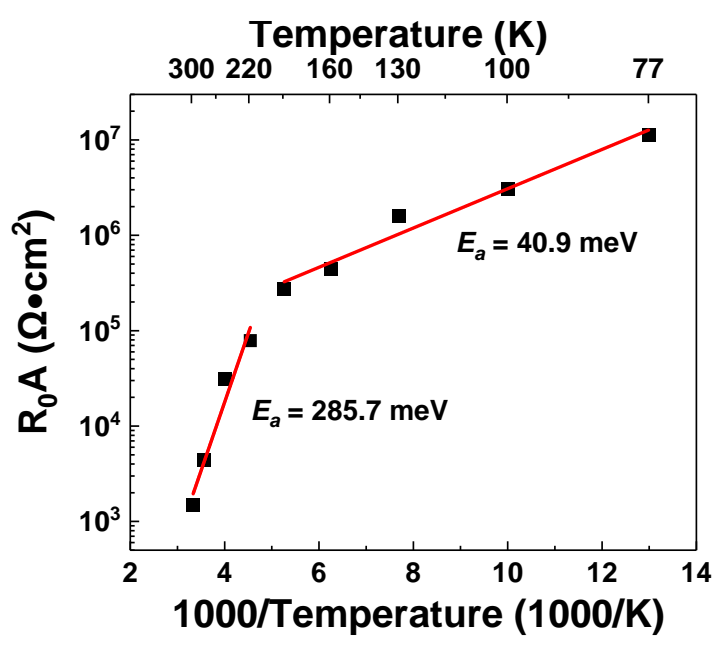

Fig. 7. Arrhenius plot of the $\mathrm{R}_{0} \mathrm{~A}$ product.

In order to further investigate the optoelectronic performances of the QD-QCD, a $130 \mu \mathrm{m}$ device was wire-bonded and mounted into a low-temperature cryostat for photoresponse measurement. The photoresponse of the device was measured in normal incidence configuration and without anti-reflection (AR) coating. The current signal was amplified by a low noise current preamplifier, and then analyzed and displayed by a fast Fourier transform (FFT) network spectrum analyzer. A Fourier transform infrared spectrometer (FTIR) was used to measure the relative photoresponse of the sample. A standard blackbody source at $700{ }^{\circ} \mathrm{C}$ with modulation frequency of $140 \mathrm{~Hz}$ was used to measure the blackbody responsivity and calibrate the relative responsivity. Fig. 8 shows the responsivity of the QD-QCD sample measured under zero bias at $77 \mathrm{~K}$ and $100 \mathrm{~K}$. The photoresponse covers the spectral range from 5 to $8 \mu \mathrm{m}$, which is slightly broader than the GaAs-based shown in our previous work [6]. That could also be an evidence of a larger inhomogeneity of the quantum dot size in Si-based sample. The peak responsivity at $\sim 6.2 \mu \mathrm{m}(200$ $\mathrm{meV}$ ) is $0.59 \mathrm{~mA} / \mathrm{W}$ and $0.39 \mathrm{~mA} / \mathrm{W}$ at $77 \mathrm{~K}$ and $100 \mathrm{~K}$, respectively, which is corresponding to inter-subband transition from level E1 to E2. As the temperature increases, the responsivity decreases. This might be attributed to the thermal backing-filling of the excited carriers to the lowest level of the extraction staircase from the QDs ground state in next cascade period (E1-E7), which could result in reduction of absorption efficiency and consequently the responsivity [3]. In addition, a decrease in photo-excited electron lifetime at higher temperature could also result in the reduction of photoresponse.

The blackbody responsivity of the SML QD-QCD sample as a function of reverse bias is plotted in Fig. 9. Again, for the comparison purposes, the blackbody responsivity of the SML QD-QCD grown on GaAs substrate is also shown in Fig. 9. Here the blackbody responsivity is defined as the ratio of output photocurrent and the input radiation power from the blackbody source [22, 23]. The blackbody responsivity of GaAs based structure is larger than that of the $\mathrm{Si}$ based device, which is 0.14 $\mathrm{mA} / \mathrm{W}$ and $0.05 \mathrm{~mA} / \mathrm{W}$ under zero bias, respectively. The corresponding peak responsivity is $1.9 \mathrm{~mA} / \mathrm{W}$ versus 
$0.59 \mathrm{~mA} / \mathrm{W}$ for the GaAs based device and Si based device respectively. It is noted that the responsivity of quantum dot QCD structure on GaAs substrate is comparable with the QD QCD on InP substrate [7]. The lower responsivity of Si based device compared to GaAs based device is due to the fact that there are more defects in $\mathrm{Si}$ based device, which capture photo-excited carriers and reduce carrier lifetime, thus, the responsivity decreased. In addition, as the reverse bias increases, the blackbody responsivity of two detectors increase slightly. This is because a larger reverse bias facilitates the relaxation of excited carrier to next period of the extraction cascade, consequently, the blackbody responsivity increases.

The Johnson noise limited detectivity $D^{*}$ can be calculated by [6]:

$$
D^{*}{ }_{j}=R_{p} \sqrt{\frac{R_{0} A}{4 k_{B} T}}
$$

where $R_{p}$ is the peak responsivity, $k_{B}$ is the Boltzmann constant, and $\mathrm{T}$ is the temperature. Johnson noise limited detectivity of the SML QD-QCD device at $77 \mathrm{~K}$ and $100 \mathrm{~K}$ were calculated and presented in Fig. 10. Even with the small responsivity, the peak detectivity reaches $3 \times 10^{10} \mathrm{~cm} \cdot \mathrm{Hz}^{1 / 2} / \mathrm{W}$ at $\sim 6.2 \mu \mathrm{m}$ and $77 \mathrm{~K}$, and it drops to $9.5 \times 10^{9} \mathrm{~cm} \cdot \mathrm{Hz}^{1 / 2} / \mathrm{W}$ when temperature increases to $100 \mathrm{~K}$. The peak detectivity of this Si-based SML QD-QCD shown in Fig. 10 is comparable to that of the SK-grown QD-QCD on GaAs substrate demonstrated in Barve's work [5].

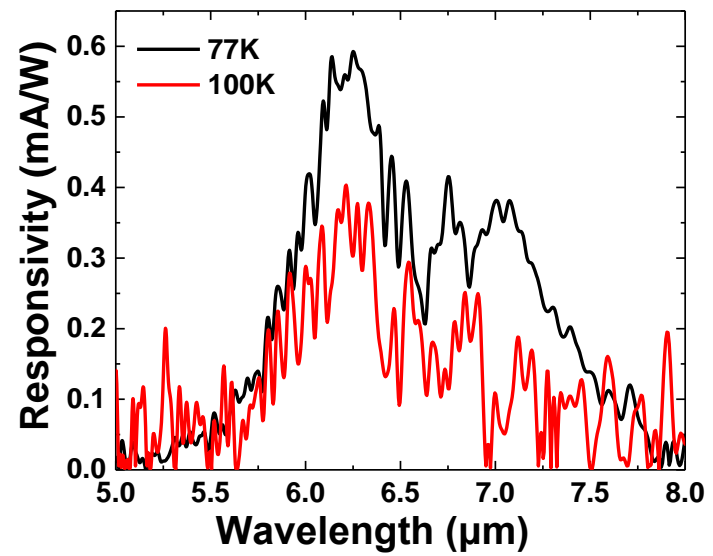

Fig. 8. Normal incident responsivity of the QD-QCD sample measured at $77 \mathrm{~K}$ and $100 \mathrm{~K}$ under zero bias.

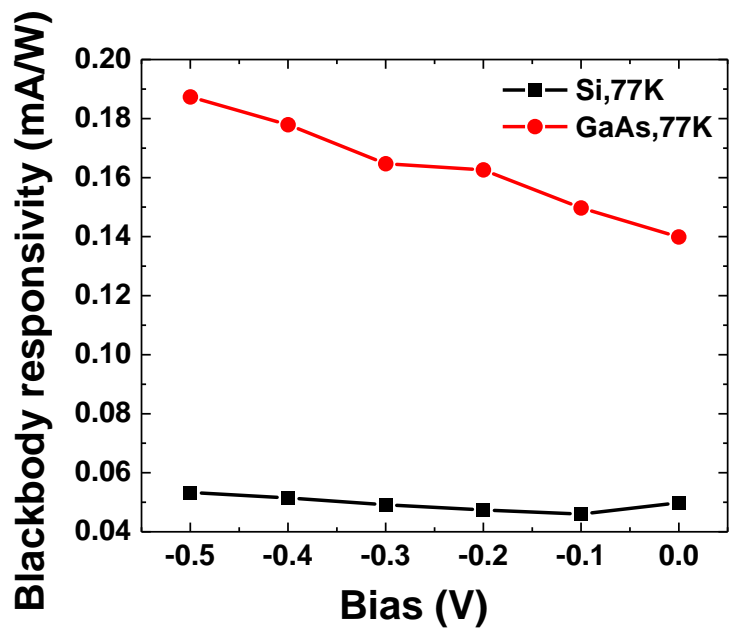

Fig. 9. Blackbody responsivity as a function of reverse bias measured from the $\mathrm{Si}$ and $\mathrm{GaAs}$ based photodetectors, respectively, at $77 \mathrm{~K}$.

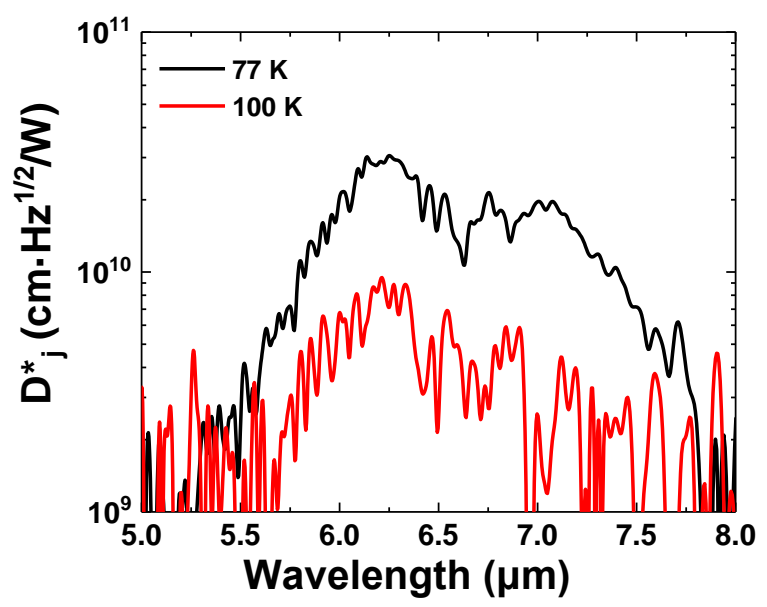

Fig. 10. Johnson noise limited detectivity of the SML QD-QCD sample calculated at $77 \mathrm{~K}$ and $100 \mathrm{~K}$ under zero bias.

\section{CONCLUSIONS}

In conclusion, we have reported a sub-monolayer quantum dot quantum cascade photodetector grown on $\mathrm{Si}$ substrate. A peak Johnson noise limited detectivity of $3 \times 10^{10} \mathrm{~cm} \cdot \mathrm{Hz}^{1 / 2} / \mathrm{W}$ under zero bias at $6.2 \mu \mathrm{m}$ at $77 \mathrm{~K}$ is achieved in this SML QD-QCD with the corresponding responsivity of $0.59 \mathrm{~mA} / \mathrm{W}$. Although the lattice mismatch between Si substrate and GaAs affects the performance of QD-QCD compared with that of the devices grown on native III-V substrate, these preliminary results show that the performance of this SMLQD-QCD directly grown on silicon substrate is very promising for future cost-effective silicon photonics application. The performance of the device can further be improved by optimizing the device structure with better design (such as using resonant tunneling transition), passivating the surface of the device, growing more defect filter layers and optimizing the MBE growth condition.

\section{REFERENCES}


[1] A. P. Ravikumar, J. De Jesus, M. C. Tamargo, and C. F. Gmachl, "High performance, room temperature, broadband II-VI quantum cascade detector," Applied Physics Letters, vol. 107, 2015.

[2] A. P. Ravikumar, D. Sivco, and C. F. Gmachl, "Wavelength independent normal incident quantum cascade detectors," Opt Express, vol. 24, pp. 25269-25276, Oct 312016.

[3] A. Harrer, B. Schwarz, S. Schuler, P. Reininger, A. Wirthmuller, H. Detz, et al., "4.3 mum quantum cascade detector in pixel configuration," Opt Express, vol. 24, pp. 17041-9, Jul 252016.

[4] L. Gendron, C. Koeniguer, X. Marcadet, and V. Berger, "Quantum cascade detectors," Infrared Physics \& Technology, vol. 47, pp. 175-181, 2005.

[5] A. V. Barve and S. Krishna, "Photovoltaic quantum dot quantum cascade infrared photodetector," Applied Physics Letters, vol. 100, 2012.

[6] J. Huang, D. Guo, W. Chen, Z. Deng, Y. Bai, T. Wu, et al., "Sub-monolayer quantum dot quantum cascade mid-infrared photodetector," Applied Physics Letters, vol. 111, 2017.

[7] X.-J. Wang, S.-Q. Zhai, N. Zhuo, J.-Q. Liu, F.-Q. Liu, S.-M. Liu, et al., "Quantum dot quantum cascade infrared photodetector," Applied Physics Letters, vol. 104, 2014.

[8] H. Kim, S.-Y. Ahn, S. Kim, G. Ryu, J. H. Kyhm, K. W. Lee, et al., "InAs/GaAs quantum dot infrared photodetector on a Si substrate by means of metal wafer bonding and epitaxial lift-off," Optics Express, vol. 25, 2017.

[9] Y. Wan, Z. Zhang, R. Chao, J. Norman, D. Jung, C. Shang, et al., "Monolithically integrated InAs/InGaAs quantum dot photodetectors on silicon substrates," Opt Express, vol. 25, pp. 27715-27723, Oct 302017.

[10] R. Soref, "Mid-infrared photonics in silicon and germanium," Nature photonics, vol. 4, pp. 495-497, 2010.

[11] A. Malik, S. Dwivedi, L. Van Landschoot, M. Muneeb, Y. Shimura, G. Lepage, et al., "Ge-on-Si and Ge-on-SOI thermo-optic phase shifters for the mid-infrared," Optics express, vol. 22, pp. 28479-28488, 2014.

[12] M. Nedeljkovic, S. Stankovic, C. J. Mitchell, A. Z. Khokhar, S. A Reynolds, D. J. Thomson, et al., "Mid-infrared thermo-optic modulators in SoI," IEEE Photon. Technol. Lett., vol. 26, pp. 1352-1355, 2014

[13] B. Chen, "Active Region Design and Gain Characteristics of InP-Based Dilute Bismide Type-II Quantum Wells for Mid-IR Lasers," IEEE Transactions on Electron Devices, vol. 64, pp. 1606-1611, 2017.

[14] B. Chen, "Optical gain analysis of GaAs-based InGaAs/GaAsSbBi type-II quantum wells lasers," Opt Express, vol. 25, pp. 25183-25192, Oct 162017

[15] B. Chen and A. Holmes, "Optical gain modeling of InP based InGaAs (N)/GaAsSb type-II quantum wells laser for mid-infrared emission," Optical and Quantum Electronics, pp. 1-8, 2013.

[16] J. Wu, Q. Jiang, S. Chen, M. Tang, Y. I. Mazur, Y. Maidaniuk, et al., "Monolithically integrated InAs/GaAs quantum dot mid-infrared photodetectors on silicon substrates," ACS Photonics, vol. 3, pp. 749-753, 2016.

[17] W. Chen, Z. Deng, D. Guo, Y. Chen, Y. I. Mazur, Y. Maidaniuk, et al., "Demonstration of InAs/InGaAs/GaAs Quantum Dots-in-a-Well Mid-Wave Infrared Photodetectors Grown on Silicon Substrate," Journal of Lightwave Technology, vol. 36, pp. 2572-2581, 2018/07/01 2018.

[18] N. Gautam, S. Myers, A. V. Barve, B. Klein, E. P. Smith, D. R. Rhiger, et al., "High operating temperature interband cascade midwave infrared detector based on type-II InAs/GaSb strained layer superlattice," Applied Physics Letters, vol. 101, 2012.

[19] A. P. Ravikumar, T. A. Garcia, J. D. Jesus, M. C. Tamargo, and C. F. Gmachl, "High detectivity short-wavelength II-VI quantum cascade detector," Applied Physics Letters, vol. 105, 2014.

[20] C. Koeniguer, G. Dubois, A. Gomez, and V. Berger, "Electronic transport in quantum cascade structures at equilibrium," Physical Review B, vol. 74, 2006.

[21] A. Gomez, N. Péré-Laperne, L.-A. de Vaulchier, C. Koeniguer, A Vasanelli, A. Nedelcu, et al., "Dark current analysis of quantum cascade detectors by magnetoresistance measurements," Physical Review B, vol. 77, 2008.

[22] B. Chen and A. L. Holmes, "InP-based short-wave infrared and midwave infrared photodiodes using a novel type-II strain-compensated quantum well absorption region," Optics letters, vol. 38, pp. 2750-2753, 2013

[23] B. Chen, W. Jiang, J. Yuan, A. L. Holmes, and B. M. Onat, "SWIR/MWIR InP-based PIN photodiodes with InGaAs/GaAsSb type-II quantum wells," IEEE Journal of Quantum Electronics, vol. 47, pp. 1244-1250, 2011. 